\title{
Charge stiffness and long-range correlation in the optically induced $\eta$-pairing state of the one-dimensional Hubbard model
}

\author{
Tatsuya Kaneko, ${ }^{1}$ Seiji Yunoki, ${ }^{2,3,4}$ and Andrew J. Millis ${ }^{1,5}$ \\ ${ }^{1}$ Department of Physics, Columbia University, New York, New York 10027, USA \\ ${ }^{2}$ Computational Condensed Matter Physics Laboratory, RIKEN Cluster for Pioneering Research (CPR), Wako, Saitama 351-0198, Japan \\ ${ }^{3}$ Computational Materials Science Research Team, RIKEN Center for Computational Science (R-CCS), Kobe, Hyogo 650-0047, Japan \\ ${ }^{4}$ Computational Quantum Matter Research Team, RIKEN Center for Emergent Matter Science (CEMS), Wako, Saitama 351-0198, Japan \\ ${ }^{5}$ Center for Computational Quantum Physics, Flatiron Institute, New York, New York 10010, USA
}

(Received 23 October 2019; revised 17 May 2020; accepted 2 July 2020; published 29 July 2020)

\begin{abstract}
We show that optical excitation of the Mott insulating phase of the one-dimensional Hubbard model can create a state possessing two of the hallmarks of superconductivity: a nonvanishing charge stiffness and longranged pairing correlation. By employing the exact diagonalization method, we find that the superposition of the $\eta$-pairing eigenstates induced by the optical pump exhibits a nonvanishing charge stiffness and a pairing correlation that decays very slowly with system size, in sharp contrast to the behavior of an ensemble of thermally excited eigenstates, which has a vanishing charge stiffness and no long-ranged pairing correlations. We show that the charge stiffness is indeed directly associated with the $\eta$-pairing correlation in the Hubbard model. Our finding demonstrates that optical pumping can actually lead to superconducting-like properties on the basis of the $\eta$-pairing states.
\end{abstract}

DOI: 10.1103/PhysRevResearch.2.032027

A fundamental goal of nonequilibrium physics is to use strong light-matter interactions to create new quantum phases [1-4]. Recent experimental observation of possible lightinduced superconductivity [5-9] has attracted much attention and stimulated many theoretical studies [10-17]. These studies focus mainly on the possibility that a light pulse can change the Hamiltonian from one with a nonsuperconducting state into one with a superconducting phase anticipated in equilibrium. In contrast, in this paper, we show that optical excitation of the Mott insulating phase of the one-dimensional (1D) Hubbard model excites the system into a state characterized by two of the hallmarks of superconductivity: a nonvanishing charge stiffness $D$ and a pairing correlation $P_{i j}=\left\langle\hat{c}_{i, \downarrow}^{\dagger} \hat{c}_{i, \uparrow}^{\dagger} \hat{c}_{j, \uparrow} \hat{c}_{j, \downarrow}\right\rangle_{i \neq j}$ that decays very slowly with system size. The components of this state are present in the spectrum but do not give rise to superconducting properties in thermal equilibrium; in other words optical excitation reveals a hidden pairing state.

Kaneko et al. [18] showed previously that optical pumping the Mott insulating phase of the Hubbard model created a state characterized by a pairing correlation $P_{i j}$, whose Fourier transform exhibited a very strong peak at the wave vector $q=\pi$, indicating that a pair density wave state was created [18]. The pair density wave state was attributed to the

Published by the American Physical Society under the terms of the Creative Commons Attribution 4.0 International license. Further distribution of this work must maintain attribution to the author(s) and the published article's title, journal citation, and DOI. preferential creation, by the nonequilibrium drive, of Yang's $\eta$-paired states [19]. Subsequent work has demonstrated that $\eta$-pairing can be induced by other protocols including injection of doublon-hole pairs [20,21] and effect of dissipation $[22,23]$. These $\eta$-pairing states are characterized by the operators $\hat{\eta}^{+}=\sum_{j}(-1)^{j} \hat{c}_{j, \downarrow}^{\dagger} \hat{c}_{j, \uparrow}^{\dagger}, \hat{\eta}^{-}=\left(\hat{\eta}^{+}\right)^{\dagger}$, and $\hat{\eta}_{z}=$ $\frac{1}{2} \sum_{j}\left(\hat{n}_{j, \uparrow}+\hat{n}_{j, \downarrow}-1\right)$, where the operators obey the standard SU(2) commutation relations and the operator $\hat{\eta}^{+}$creates in effect a paired state with a staggered pairing amplitude [19,24]. Since the Hubbard Hamiltonian commutes with the operator $\hat{\eta}^{2}=\frac{1}{2}\left(\hat{\eta}^{+} \hat{\eta}^{-}+\hat{\eta}^{-} \hat{\eta}^{+}\right)+\hat{\eta}_{z}^{2}$, Hubbard eigenstates are simultaneously eigenstates of $\hat{\eta}^{2}$, and Yang has shown that a Hubbard eigenstate with a nonzero value of $\left\langle\hat{\eta}^{2}\right\rangle$ has long-ranged pairing correlations $\left\langle\eta_{i}^{+} \eta_{j}^{-}\right\rangle_{i \neq j}=$ $(-1)^{i+j}\left\langle\hat{c}_{i, \downarrow}^{\dagger} \hat{c}_{i, \uparrow}^{\dagger} \hat{c}_{j, \uparrow} \hat{c}_{j, \downarrow}\right\rangle[19]$.

While previous work reveals that the pump electric field induces $\eta$-pairing states [18], actual superconducting properties were not established. In this paper, we employ an eigenstate analysis and systematic finite-size scaling to show that the photoinduced $\eta$-pairing state has nonzero charge stiffness and long-ranged pairing correlations, in contrast, for example, to any thermodynamic ensemble average over states at half-filling, which would yield an ensemble with no charge stiffness [25-28]. We also determine the optimal pump profile for the $\eta$ pairing and clarify its system size dependence.

We here study the 1D Hubbard model with the nearest neighbor hopping $t_{h}$ and on-site interaction $U>0$ :

$$
\hat{\mathcal{H}}=-t_{h} \sum_{j=1}^{L} \sum_{\sigma}\left(\hat{c}_{j, \sigma}^{\dagger} \hat{c}_{j+1, \sigma}+\text { H.c. }\right)+U \sum_{j=1}^{L} \hat{n}_{j, \uparrow} \hat{n}_{j, \downarrow},
$$




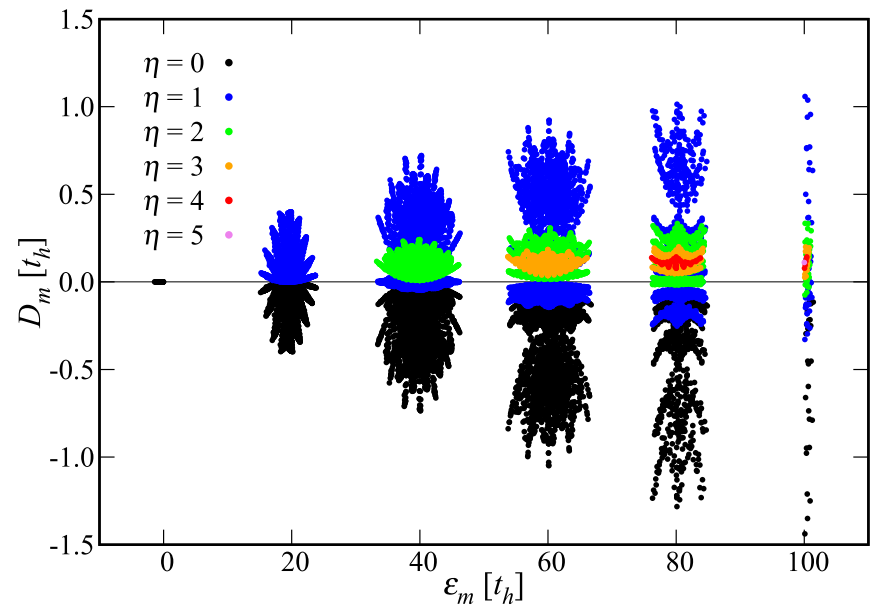

FIG. 1. Charge stiffness $D_{m}$ of the eigenstates $\left|\psi_{m}\right\rangle$ (eigenenergies $\varepsilon_{m}$ ) in the half-filled Hubbard chain calculated by the ED method for $L=10\left(N_{\uparrow}=N_{\downarrow}=5\right)$ at $U=20 t_{h}$. The colors of the points indicate the values of $\eta$.

where $\hat{c}_{j, \sigma}\left(\hat{c}_{j, \sigma}^{\dagger}\right)$ is the annihilation (creation) operator for an electron at site $j$ with spin $\sigma(=\uparrow, \downarrow)$ and $\hat{n}_{j, \sigma}=\hat{c}_{j, \sigma}^{\dagger} \hat{c}_{j, \sigma}$. We specialize to the half-filled case with the number of electrons in each spin channel, $N_{\sigma}=L / 2$ (number of sites $L$ is taken to be even). Since $\left[\hat{\mathcal{H}}, \hat{\eta}^{2}\right]=\left[\hat{\mathcal{H}}, \hat{\eta}_{z}\right]=0$, any eigenstate of $\hat{\mathcal{H}}$ is also the eigenstate $\left|\eta, \eta_{z}\right\rangle$ of $\hat{\eta}^{2}$ and $\hat{\eta}_{z}$ with the eigenvalues $\eta(\eta+1)$ and $\eta_{z}$, respectively. At half-filling, the allowed eigenvalues $\left|\eta, \eta_{z}\right\rangle$ are $\eta=0,1,2, \ldots, L / 2$ and $\eta_{z}=0$.

A time-dependent external field $A(t)$ is introduced via the Peierls substitution $t_{h} \hat{c}_{j, \sigma}^{\dagger} \hat{c}_{j+1, \sigma} \rightarrow t_{h} e^{i A(t)} \hat{c}_{j, \sigma}^{\dagger} \hat{c}_{j+1, \sigma}$. We use a pump pulse given as $A(t)=A_{0} e^{-\left(t-t_{0}\right)^{2} /\left(2 \sigma_{p}^{2}\right)} \cos \left[\omega_{p}\left(t-t_{0}\right)\right]$ with amplitude $A_{0}$, frequency $\omega_{p}$, and pulse width $\sigma_{p}$ centered at time $t_{0}(>0)$ [29]. We assume that for $t=0$ the system is in the Mott insulating ground state and evolve the state forward in time using $\hat{\mathcal{H}}(t)$, which is $\hat{\mathcal{H}}$ with the time-dependent hopping. We employ the time-dependent exact diagonalization (ED) method [30,31] for a finite-size cluster with periodic boundary conditions (PBC), and the state at time $t$ is indicated by $|\Psi(t)\rangle$. For $t-t_{0} \gg \sigma_{p}$, the resulting state is projected onto the eigenstates $\left|\psi_{m}\right\rangle$ (eigenenergies $\varepsilon_{m}$ ) of the unperturbed Hubbard model, obtained by full (exact) diagonalization. For each eigenstate, we directly calculate the $\eta$-pairing eigenvalue $\eta(\eta+1)$. We compute the charge stiffness [32] for each eigenstate $\left|\psi_{m}\right\rangle$ from

$$
D_{m}=\left.\frac{L}{2} \frac{\partial^{2} \varepsilon_{m}(\Phi)}{\partial \Phi^{2}}\right|_{\Phi=0},
$$

with twisted boundary conditions (TBC), where the phase $\Phi$ is introduced via a vector potential $A_{\text {twist }}=\Phi / L[33,34]$. Details of the method and TBC are given in the Supplemental Material [35].

Figure 1 shows the calculated stiffnesses $D_{m}$ for all eigenstates in the half-filled Hubbard chain at a large value of the interaction $U$. The eigenstates are grouped into sectors corresponding to different numbers of doubly occupied sites. Significantly, most of $D_{m}$ for the $\eta$-pairing eigenstates $(\eta>0)$ are positive, but most of $D_{m}$ for the non- $\eta$-pairing eigenstates
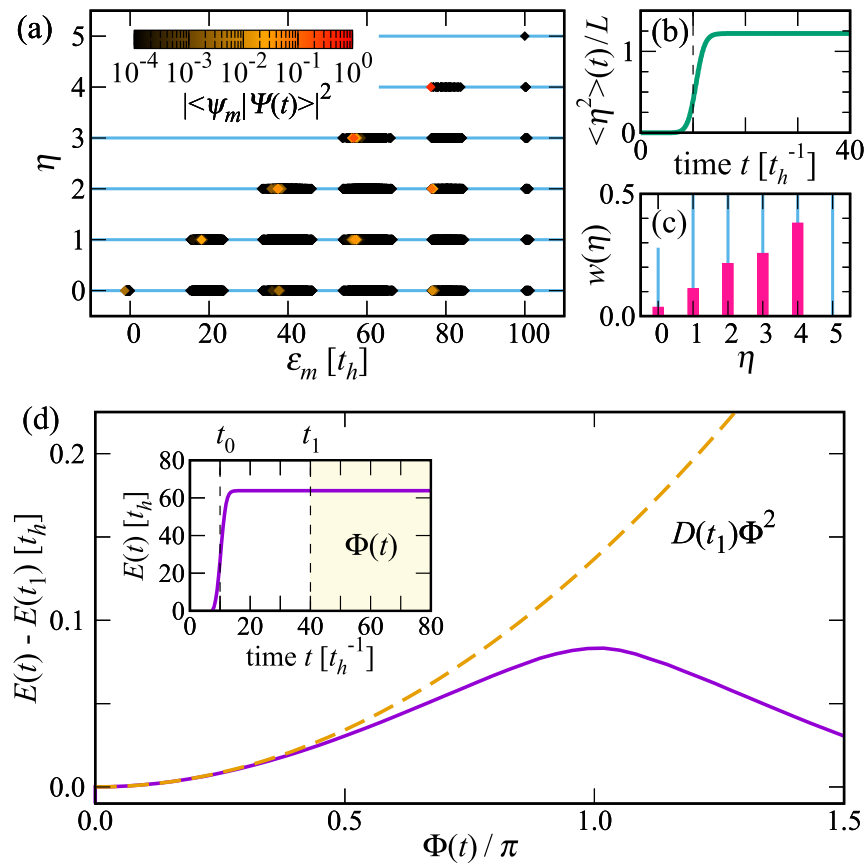

FIG. 2. (a) All eigenenergies $\varepsilon_{m}$ and eigenvalues $\eta$ for the eigenstates $\left|\psi_{m}\right\rangle$ of the half-filled Hubbard Hamiltonian $\hat{\mathcal{H}}$ at $U=20 t_{h}$ and $L=10\left(N_{\uparrow}=N_{\downarrow}=5\right)$ with PBC. The color of each point indicates the weight $\left|\left\langle\psi_{m} \mid \Psi(t)\right\rangle\right|^{2}$ of the eigenstate $\left|\psi_{m}\right\rangle$ in the photoinduced state $|\Psi(t)\rangle$ at $t=40 / t_{h}$ for $A(t)$ with $A_{0}=0.3$, $\omega_{p}=19.36 t_{h}, \sigma_{p}=2 / t_{h}$, and $t_{0}=10 / t_{h}$. (b) Time evolution of $\left\langle\hat{\eta}^{2}\right\rangle(t) / L=\left\langle\Psi(t)\left|\hat{\eta}^{2}\right| \Psi(t)\right\rangle / L$ for the same model parameters in (a). (c) Total weight $w(\eta)$ of $\left|\left\langle\psi_{m} \mid \Psi(t)\right\rangle\right|^{2}$ over the states $\left|\psi_{m}\right\rangle$ with the same number $\eta$ in (a). Note that $\sum_{\eta=0}^{L / 2} w(\eta)=1$. (d) Time evolution of the energy $E(t)=\langle\Psi(t)|\hat{\mathcal{H}}(t)| \Psi(t)\rangle$ under the timedependent flux $\Phi(t)=\theta\left(t-t_{1}\right) \times\left[\delta \Phi \cdot\left(t-t_{1}\right)\right]$ applied after the pulse irradiation $\left(t_{1}>t_{0}\right)$. The dashed line indicates $\Delta E(\Phi)=$ $D\left(t_{1}\right) \Phi^{2} / L$ with the charge stiffness $D(t)$ at $t=t_{1}$ evaluated by $D(t)=\sum_{m} D_{m}\left|\left\langle\psi_{m} \mid \Psi(t)\right\rangle\right|^{2}$ (see the text). The inset shows $E(t)$ in the whole energy scale. The results are calculated using the ED method with $\delta \Phi=0.5 \times 10^{-3}$ and $t_{1}=40 / t_{h}$ in $\Phi(t)$.

$(\eta=0)$ are negative. The sum of $D_{m}$ over all eigenstates is zero, because $S(\eta)=\sum_{m} D_{m}(\eta)$, the sum of the charge stiffness of all eigenstates with the same $\eta$, satisfies $S(\eta=$ $0)+\sum_{\eta=1}^{L / 2} S(\eta)=0$ [35]. This implies that the thermal ensemble at infinite temperature cannot have perfect conducting behavior. We find numerically that the sum of $D_{m}$ over all eigenstates in a given double occupancy sector is also zero, and the sum of $D_{m}$ over all eigenstates within a given small energy range is close to zero. This strongly suggests that the thermal average of charge stiffness is zero in equilibrium at any temperature, as theoretically expected [25-28]. To obtain $D>0$ in the half-filled Hubbard chain, one must prepare an ensemble in which $\eta$-pairing $(\eta>0)$ eigenstates have larger weight than $\eta=0$ eigenstates. We next show that photoexcitation produces just such an ensemble.

Before showing $D(t)$, we review the photoinduced state $|\Psi(t)\rangle$ and its weight distribution. As shown in Fig. 2(b), the external pulse $A(t)$ induces an $\eta$-pairing correlation $\left\langle\hat{\eta}^{2}\right\rangle(t)=\left\langle\Psi(t)\left|\hat{\eta}^{2}\right| \Psi(t)\right\rangle=\left\langle\Psi(t)\left|\hat{\eta}^{+} \hat{\eta}^{-}\right| \Psi(t)\right\rangle$ at half-filling, corresponding to the enhancement of the superconducting 
correlation at momentum $q=\pi$ shown in Ref. [18]. Figure. 2(a) shows the weight distribution of the eigenstates $\left|\psi_{m}\right\rangle$ in the photoinduced state $|\Psi(t)\rangle$, where the color of each point indicates the weight $\left|\left\langle\psi_{m} \mid \Psi(t)\right\rangle\right|^{2}$ and the total weight is shown as a function of $\eta$ in Fig. 2(c). These results clearly show that photoexcitation preferentially induces eigenstates $\left|\psi_{m}\right\rangle$ with $\eta>0$, explaining the large value of $\left\langle\hat{\eta}^{2}\right\rangle(t)$ observed in the photoinduced state $|\Psi(t)\rangle$. This photoinduced nonthermal distribution implies $D(t)>0$.

To verify the stiffness $D(t)>0$ in this photoinduced state $|\Psi(t)\rangle$, we apply the time-dependent flux $A(t)=\Phi(t) / L$, given by $\Phi(t)=\theta\left(t-t_{1}\right) \times\left[\delta \Phi \cdot\left(t-t_{1}\right)\right]$, beginning at time $t_{1}$ long after the pump pulse $\left(t_{1}-t_{0} \gg \sigma_{p}\right)$, where $\theta(t)$ is the Heaviside step function and $\Phi(t)$ increases linearly in time with slope $\delta \Phi$, corresponding to an electric field $\frac{\partial A(t)}{\partial t} \propto \delta \Phi$. To estimate the stiffness in the photoinduced state $|\Psi(t)\rangle$, we compute the energy $E(t)=\langle\Psi(t)|\hat{\mathcal{H}}(t)| \Psi(t)\rangle$ under the time-dependent flux $\Phi(t)$. As shown in Fig. 2(d), the curvature of $E(t)$ is positive with respect to $\Phi(t)$, indicating $D(t)>0$. To identify the curvature of the energy $E(t)$ at $\Phi=0$, we should notice that the charge stiffness $D(t)=\sum_{m}\left|c_{m}(t)\right|^{2} D_{m}$ can also be evaluated directly from the weight $\left|c_{m}(t)\right|^{2}=$ $\left|\left\langle\psi_{m} \mid \Psi(t)\right\rangle\right|^{2}$ in the photoinduced state $|\Psi(t)\rangle$. Comparing with $\Delta E(\Phi)=D\left(t_{1}\right) \Phi^{2} / L$, the energy curve $E(t)$ at $\Phi(t) \sim$ 0 is perfectly fitted by the stiffness $D\left(t_{1}\right)$ evaluated from the photoinduced weight distribution. Therefore, the photoinduced state $|\Psi(t)\rangle$ has a stiffness $D(t)>0$.

The above results demonstrate an association between a nonthermal distribution of states with $\left\langle\hat{\eta}^{2}\right\rangle \neq 0$ and a nonvanishing charge stiffness. We now show that these two factors are also associated with long-ranged $\eta$-pairing correlation. First, we see this association in Yang's maximally $\eta$-paired state $\left|\phi_{N_{\eta}}\right\rangle \propto\left(\hat{\eta}^{+}\right)^{N_{\eta}}|0\rangle$ generated from the vacuum $|0\rangle$ [19]. For this state, Yang showed that the $\eta$-pairing correlation is distance independent and of infinite range with $\left\langle\phi_{N_{\eta}}\left|\hat{\eta}_{i}^{+} \hat{\eta}_{j}^{-}\right| \phi_{N_{\eta}}\right\rangle_{i \neq j}=\frac{N_{\eta}\left(L-N_{\eta}\right)}{L(L-1)}$ [19]. Here we find that the charge stiffness $D_{\eta}$ for Yang's $\eta$-pairing state $\left|\phi_{N_{\eta}}\right\rangle$ satisfies $D_{\eta}=4 J_{\text {ex }}\left\langle\phi_{N_{\eta}}\left|\hat{\eta}_{i}^{+} \hat{\eta}_{j}^{-}\right| \phi_{N_{\eta}}\right\rangle_{i \neq j}>0$ with the exchange interaction $J_{\mathrm{ex}}=2 t_{h}^{2} / U$ (see details in the Supplemental Material [35]), which directly associates the charge stiffness with the long-ranged pairing correlation.

Our numerical evidence strongly suggests that this association is valid beyond Yang's $\eta$-pairing state. To discuss this, let us review the ingredients of $\left\langle\hat{\eta}^{2}\right\rangle$. At half-filling $\left(\eta_{z}=0\right)$, the algebra of $\eta$ operators implies

$$
\left\langle\hat{\eta}^{2}\right\rangle=L n_{d}+\sum_{i \neq j}\left\langle\hat{\eta}_{i}^{+} \hat{\eta}_{j}^{-}\right\rangle
$$

with the double occupancy $n_{d}=\frac{1}{L} \sum_{j}\left\langle\hat{n}_{j, \uparrow} \hat{n}_{j, \downarrow}\right\rangle$. From the analysis of the eigenstates, we can show $\left\langle\hat{\eta}^{2}\right\rangle\left(n_{d}\right) \equiv$ $\frac{1}{\mathcal{N}_{n_{d}}} \sum_{m}\left\langle\psi_{m}\left|\hat{\eta}^{2}\right| \psi_{m}\right\rangle_{n_{d}}=L n_{d}$ in each double occupancy $\left(n_{d}\right)$ sector, where $\mathcal{N}_{n_{d}}$ is the number of the eigenstates and the suffix $n_{d}$ indicates the eigenstate within the $n_{d}$ sector (see the Supplemental Material [35]). We can also show that the average of $\left\langle\psi_{m}\left|\hat{\eta}^{2}\right| \psi_{m}\right\rangle$ over all Hubbard eigenstates at half-filling is $\left\langle\hat{\eta}^{2}\right\rangle_{\text {avr. }} / L=0.25$, which is same with the double occupancy $n_{d}=0.25$ at infinite temperature. Comparing with Eq. (3), these re-

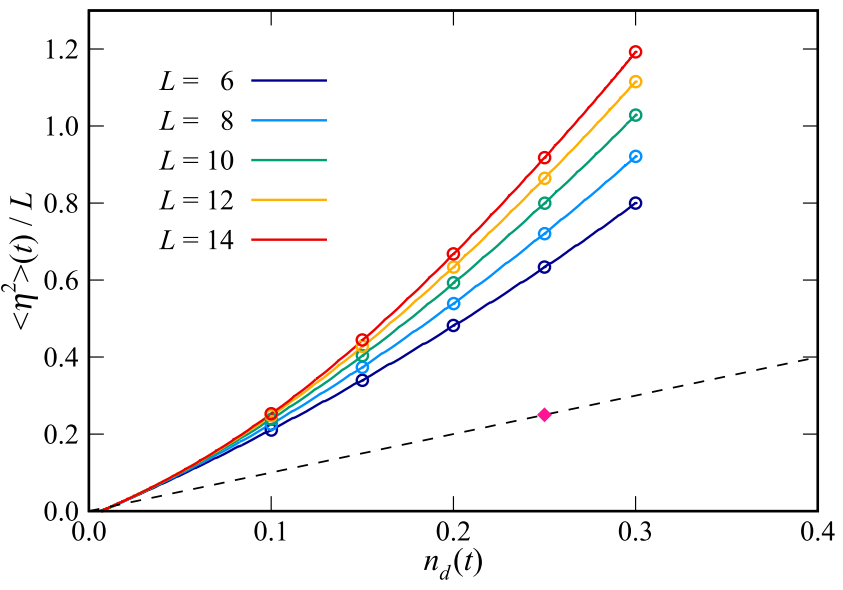

FIG. 3. $\left\langle\hat{\eta}^{2}\right\rangle(t) / L$ as the function of $n_{d}(t)$ in the half-filled Hubbard chain at $U=20 t_{h}$ with $\omega_{p} / t_{h}=18.68,19.11,19.36,19.54$, and 19.66 for $L=6,8,10,12$, and 14, respectively. The dashed line is $\left\langle\hat{\eta}^{2}\right\rangle\left(n_{d}\right) / L=n_{d}$. The diamond indicates $\left\langle\hat{\eta}^{2}\right\rangle / L=n_{d}=0.25$, which is the average of $\left\langle\psi_{m}\left|\hat{\eta}^{2}\right| \psi_{m}\right\rangle$ over all Hubbard eigenstates at half-filling $\left(N_{\uparrow}=N_{\downarrow}=L / 2\right)$. The results are calculated by the ED method under PBC with $A_{0}=0.3, \sigma_{p}=2 / t_{h}$, and $t_{0}=10 / t_{h}$ in $A(t)$.

lations strongly suggest that a thermal distribution of the eigenstates has no long-range $\eta$-pairing correlation. However, we find for the optically generated state $|\Psi(t)\rangle$ that $\left\langle\hat{\eta}^{2}\right\rangle>L n_{d}$ [see, e.g., Fig. 2(b), where $\left\langle\hat{\eta}^{2}\right\rangle(t) / L>1$ ], which implies contributions from nonlocal pairing correlations $\left\langle\hat{\eta}_{i}^{+} \hat{\eta}_{j}^{-}\right\rangle_{i \neq j}$ in Eq. (3).

We now analyze the spatial correlations and finite size effects in the photoinduced state. One trivial finite size effect is a weak size dependence of the optimal photoexcitation frequency $\omega_{p}$. For each system size, we calculate $\left\langle\hat{\eta}^{2}\right\rangle(t)$ with different $\omega_{p}$ (see the Supplemental Material [35]). Here we present results obtained at the optimal $\omega_{p}$ for each size. We represent the amount of optical excitation by the induced double occupancy in Fig. 3, by plotting $\left\langle\hat{\eta}^{2}\right\rangle(t) / L$ as a function of $n_{d}(t)=\frac{1}{L} \sum_{j}\left\langle\Psi(t)\left|\hat{n}_{j, \uparrow} \hat{n}_{j, \downarrow}\right| \Psi(t)\right\rangle$. Note that here we consider a fixed pump strength $A_{0}$, which produces time-dependent $n_{d}(t)$ and $\left\langle\hat{\eta}^{2}\right\rangle(t)$. Equivalent results could be obtained by $A_{0}$ dependence considering the long-time limits of $n_{d}(t)$ and $\left\langle\hat{\eta}^{2}\right\rangle(t)$ (see the Supplemental Material [35]). Figure 3 reveals two important results: $\left\langle\hat{\eta}^{2}\right\rangle(t) / L$ under photoexcitation is systematically greater than $n_{d}$ (dashed line), indicating that the $\left\langle\hat{\eta}_{i}^{+} \hat{\eta}_{j}^{-}\right\rangle_{i \neq j}$ term in Eq. (3) is nonzero, and the difference from $n_{d}$ increases with increasing system size $L$. Examination of Eq. (3) indicates that this increase must correspond to the development of long-range correlation. In comparison with an average with thermal distribution of the eigenstates, where $\left\langle\hat{\eta}^{2}\right\rangle / L \sim n_{d}$ and $D \sim 0$ are expected, $\left\langle\hat{\eta}^{2}\right\rangle(t) / L>n_{d}(t)$ in Fig. 3 implies a nonthermal distribution induced by optically preferential $\eta$-pairing states, which gives rise to a nonvanishing charge stiffness $D(t)>0$ (see, e.g., Fig. 2).

To further understand the pairing correlation, we define the quantity

$$
P_{i \neq j}^{(\eta)}(t)=\frac{1}{L^{2}} \sum_{i \neq j}\left\langle\Psi(t)\left|\hat{\eta}_{i}^{+} \hat{\eta}_{j}^{-}\right| \Psi(t)\right\rangle
$$




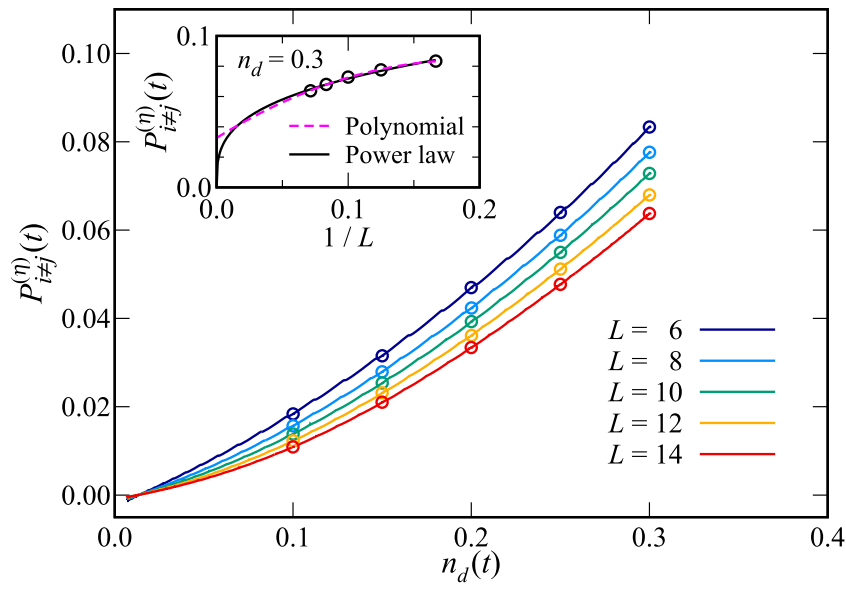

FIG. 4. Time-dependent $\eta$-pairing correlation $P_{i \neq j}^{(\eta)}(t)$ as the function of the double occupancy $n_{d}(t)$ in the half-filled Hubbard chain at $U=20 t_{h}$ with $\omega_{p} / t_{h}=18.68,19.11,19.36,19.54$, and 19.66 for $L=6,8,10,12$, and 14, respectively. Inset: Size dependence of $P_{i \neq j}^{(\eta)}(t)$ at $n_{d}(t)=0.3$. The dashed and solid lines are polynomial and power-law fittings, respectively. The results are calculated by the ED method under PBC with $A_{0}=0.3, \sigma_{p}=2 / t_{h}$, and $t_{0}=10 / t_{h}$ in $A(t)$.

When long-ranged $\eta$-pairing correlation is formed, $P_{i \neq j}^{(\eta)}(t)$ remains nonzero with increasing system size $L$, corresponding to $\left\langle\hat{\eta}^{2}\right\rangle \sim \sum_{i \neq j}\left\langle\hat{\eta}_{i}^{+} \hat{\eta}_{j}^{-}\right\rangle \propto L^{2}$. For Yang's $\eta$-pairing state $\left|\phi_{\eta}\right\rangle$, $P_{i \neq j}^{(\eta)}=0.25$ at $n_{d}=0.5$ regardless of the system size. In Fig. 4, we show $P_{i \neq j}^{(\eta)}(t)$ with the different system sizes $L$. We see for the optically created state that the magnitude is $P_{i \neq j}^{(\eta)}(t) \sim 0.07$ at $n_{d}(t)=0.3$, which is comparable to the value in Yang's maximally $\eta$-paired state. The value of $P_{i \neq j}^{(\eta)}(t)$ varies slowly with system size. The inset of Fig. 4 shows the $L$ dependence of $P_{i \neq j}^{(\eta)}(t)$ at $n_{d}(t)=0.3$. While the range of system sizes accessible to us is too small to make a definitive statement, the results are consistent with either a nonzero extrapolation to the $L \rightarrow \infty$ limit or $P_{i \neq j}^{(\eta)} \propto L^{-\alpha}$ with $\alpha \sim$ 0.3 corresponding to very slowly decaying power-law pairing correlation (quasi-long-range order).

Finally, we comment on the pulse width $\sigma_{p}$ dependence. Since a high-temperature ensemble is expected in the limit of $\sigma_{p} \rightarrow \infty$ at $\omega_{p} \sim U$ [36], there must be an optimal value of $\sigma_{p}$ for the enhancement of the $\eta$-pairing correlation. Figure 5 shows the $\sigma_{p}$ dependence of $\left\langle\hat{\eta}^{2}\right\rangle(t) / L$ computed at a long time after the optical pump. The optimal pump width and the maximal value of $\left\langle\hat{\eta}^{2}\right\rangle(t) / L$ increase with increasing $L$, which is consistent with the idea that the pump produces a state with long-ranged correlations.

In conclusion, we have shown from finite system numerics along with a scaling analysis of the system size dependence

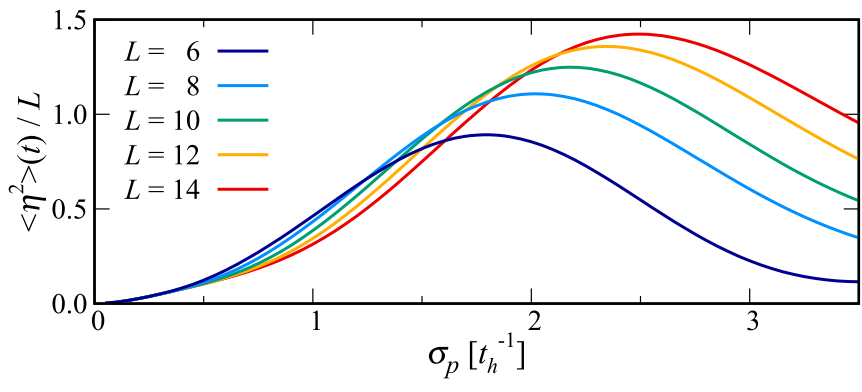

FIG. 5. Dependence of $\left\langle\hat{\eta}^{2}\right\rangle(t) / L$ on pump width $\sigma_{p}$, computed for the half-filled Hubbard chain using the ED method with PBC and $U=20 t_{h}$ at $t=10 \sigma_{p}+10 / t_{h}$ after the pump maximum. The pump frequencies are $\omega_{p} / t_{h}=18.68,19.11,19.36,19.54$, and 19.66 for $L=6,8,10,12$, and 14 , respectively, and the other pump parameters are $A_{0}=0.3$ and $t_{0}=5 \sigma_{p}$.

that optical excitation of the 1D Hubbard model creates a state possessing two of the hallmarks of superconductivity: a nonvanishing charge stiffness and long-ranged pairing correlation. The fundamental reason is that optical excitation preferentially creates $\eta$-pairing states, which as we have shown here via an eigenstate analysis have a positive stiffness with typical values of $D \sim J_{\mathrm{ex}}=2 t_{h}^{2} / U$. This work extends the previous study [18] showing that optical excitation can induce $\eta$-pairing correlations by demonstrating that the nonequilibrium ensemble created by the drive in fact has superconducting properties.

While the 1D Hubbard model we used here is in several respects a highly simplified description of real materials, it can be realized in cold atomic gasses, and our results provide predictions for experiments in these systems. But, more fundamentally, we believe that our results are important because they provide an existence proof that nonequilibrium drive can create a state with superconducting properties in an originally nonsuperconducting system. Our work provides new understanding of the qualitative properties of light-induced superconductivity, and may serve as a base for future research including both a more detailed examination of the properties of the light-induced superconducting state and extensions to higher dimensions and richer models [37].

The authors thank S. Ejima, D. Golež, and T. Shirakawa for fruitful discussion. This was supported in part by Grantsin-Aid for Scientific Research from JSPS (Projects No. JP18K13509, No. JP18H01183, and No. JP20H01849) of Japan. A.J.M. was supported by the Basic Energy Sciences program of the U.S. Department of Energy under Grant No. DE-SC0018218. T.K. was supported by the JSPS Overseas Research Fellowship.
[1] Y. Tokura, J. Phys. Soc. Jpn. 75, 011001 (2006).

[2] D. N. Basov, R. D. Averitt, and D. Hsieh, Nat. Mater. 16, 1077 (2017).

[3] T. Oka and S. Kitamura, Annu. Rev. Condens. Matter Phys. 10, 387 (2019).
[4] S. Ishihara, J. Phys. Soc. Jpn. 88, 072001 (2019).

[5] D. Fausti, R. I. Tobey, N. Dean, S. Kaiser, A. Dienst, M. C. Hoffmann, S. Pyon, T. Takayama, H. Takagi, and A. Cavalleri, Science 331, 189 (2011). 
[6] W. Hu, S. Kaiser, D. Nicoletti, C. R. Hunt, I. Gierz, M. C. Hoffmann, M. Le Tacon, T. Loew, B. Keimer, and A. Cavalleri, Nat. Mater. 13, 705 (2014)

[7] S. Kaiser, C. R. Hunt, D. Nicoletti, W. Hu, I. Gierz, H. Y. Liu, M. Le Tacon, T. Loew, D. Haug, B. Keimer, and A. Cavalleri, Phys. Rev. B 89, 184516 (2014).

[8] M. Mitrano, A. Cantaluppi, D. Nicoletti, S. Kaiser, A. Perucchi, S. Lupi, P. Di Pietro, D. Pontiroli, M. Riccò, S. R. Clark, D. Jaksch, and A. Cavalleri, Nature (London) 530, 461 (2016).

[9] A. Cantaluppi, M. Buzzi, G. Jotzu, D. Nicoletti, M. Mitrano, D. Pontiroli, M. Riccò, A. Perucchi, P. Di Pietro, and A. Cavalleri, Nat. Phys. 14, 837 (2018).

[10] S. J. Denny, S. R. Clark, Y. Laplace, A. Cavalleri, and D. Jaksch, Phys. Rev. Lett. 114, 137001 (2015).

[11] J.-i. Okamoto, A. Cavalleri, and L. Mathey, Phys. Rev. Lett. 117, 227001 (2016).

[12] M. A. Sentef, A. F. Kemper, A. Georges, and C. Kollath, Phys. Rev. B 93, 144506 (2016).

[13] M. Knap, M. Babadi, G. Refael, I. Martin, and E. Demler, Phys. Rev. B 94, 214504 (2016).

[14] D. M. Kennes, E. Y. Wilner, D. R. Reichman, and A. J. Millis, Nat. Phys. 13, 479 (2017).

[15] K. Ido, T. Ohgoe, and M. Imada, Sci. Adv. 3, e1700718 (2017).

[16] Y. Murakami, N. Tsuji, M. Eckstein, and P. Werner, Phys. Rev. B 96, 045125 (2017)

[17] G. Mazza and A. Georges, Phys. Rev. B 96, 064515 (2017).

[18] T. Kaneko, T. Shirakawa, S. Sorella, and S. Yunoki, Phys. Rev. Lett. 122, 077002 (2019).

[19] C. N. Yang, Phys. Rev. Lett. 63, 2144 (1989).

[20] P. Werner, J. Li, D. Golež, and M. Eckstein, Phys. Rev. B 100, 155130 (2019).

[21] J. Li, D. Golež, P. Werner, and M. Eckstein, arXiv:1908.08693.

[22] J. Tindall, B. Buča, J. R. Coulthard, and D. Jaksch, Phys. Rev. Lett. 123, 030603 (2019).

[23] F. Peronaci, O. Parcollet, and M. Schiró, Phys. Rev. B 101, 161101(R) (2020)

[24] F. H. Essler, H. Frahm, F. Göhmann, A. Klümper, and V. E. Korepin, The One-Dimensional Hubbard Model (Cambridge University Press, Cambridge, 2005).
[25] J. Carmelo, S.-J. Gu, and P. Sacramento, Ann. Phys. (NY) 339, 484 (2013).

[26] C. Karrasch, D. M. Kennes, and J. E. Moore, Phys. Rev. B 90, 155104 (2014).

[27] F. Jin, R. Steinigeweg, F. Heidrich-Meisner, K. Michielsen, and H. De Raedt, Phys. Rev. B 92, 205103 (2015).

[28] J. Carmelo, S. Nemati, and T. Prosen, Nucl. Phys. B 930, 418 (2018).

[29] The light velocity $c$, the elementary charge $e$, the Planck constant $\hbar$, and the lattice constant are set to 1 . The hopping $t_{h}\left(t_{h}^{-1}\right)$ is a unit of energy (time).

[30] T. J. Park and J. Light, J. Chem. Phys. 85, 5870 (1986).

[31] N. Mohankumar and S. M. Auerbach, Comput. Phys. Commun. 175, 473 (2006).

[32] The charge stiffness $D$ is the coefficient of a delta-function term in the optical response: $\operatorname{Re}[\sigma(\omega)]=2 \pi D \delta(\omega)+\sigma_{\text {reg }}(\omega)$, and $D>0$ implies dissipationless charge transport.

[33] W. Kohn, Phys. Rev. 115, 1460 (1959).

[34] H. Castella, X. Zotos, and P. Prelovšek, Phys. Rev. Lett. 74, 972 (1995).

[35] See Supplemental Material at http://link.aps.org/supplemental/ 10.1103/PhysRevResearch.2.032027 for details of the numerical method, derivations of the equations, and supplemental numerical results as well as Refs. [38-46].

[36] A. Herrmann, Y. Murakami, M. Eckstein, and P. Werner, Europhys. Lett. 120, 57001 (2018).

[37] T. Shirakawa, S. Miyakoshi, and S. Yunoki, Phys. Rev. B 101, 174307 (2020).

[38] H. Hashimoto and S. Ishihara, Phys. Rev. B 93, 165133 (2016).

[39] A. Nishino and T. Deguchi, Nucl. Phys. B 688, 266 (2004).

[40] D. J. Scalapino, S. R. White, and S. Zhang, Phys. Rev. B 47, 7995 (1993).

[41] R. Resta, J. Phys.: Condens. Matter 30, 414001 (2018).

[42] N. Byers and C. N. Yang, Phys. Rev. Lett. 7, 46 (1961).

[43] R. Fujiuchi, T. Kaneko, Y. Ohta, and S. Yunoki, Phys. Rev. B 100, 045121 (2019).

[44] M. Takahashi, Prog. Theor. Phys. 46, 401 (1971).

[45] F. H. L. Essler, V. E. Korepin, and K. Schoutens, Phys. Rev. Lett. 67, 3848 (1991).

[46] F. H. Essler, V. E. Korepin, and K. Schoutens, Nucl. Phys. B 372, 559 (1992). 